\title{
Accuracy of Consumer Wearable Heart Rate Measurement During an Ecologically Valid 24-Hour Period: Intraindividual Validation Study
}

\author{
Benjamin W Nelson ${ }^{1,2}$, MS; Nicholas B Allen ${ }^{1,2}, \mathrm{PhD}$ \\ ${ }^{1}$ Department of Psychology, University of Oregon, Eugene, OR, United States \\ ${ }^{2}$ Center for Digital Mental Health, University of Oregon, Eugene, OR, United States
}

Corresponding Author:

Benjamin W Nelson, MS

Department of Psychology

University of Oregon

1227 University Street

Eugene, OR, 97403

United States

Phone: 13108014595

Email: bwn@uoregon.edu

\section{Abstract}

Background: Wrist-worn smart watches and fitness monitors (ie, wearables) have become widely adopted by consumers and are gaining increased attention from researchers for their potential contribution to naturalistic digital measurement of health in a scalable, mobile, and unobtrusive way. Various studies have examined the accuracy of these devices in controlled laboratory settings (eg, treadmill and stationary bike); however, no studies have investigated the heart rate accuracy of wearables during a continuous and ecologically valid 24-hour period of actual consumer device use conditions.

Objective: The aim of this study was to determine the heart rate accuracy of 2 popular wearable devices, the Apple Watch 3 and Fitbit Charge 2, as compared with the gold standard reference method, an ambulatory electrocardiogram (ECG), during consumer device use conditions in an individual. Data were collected across 5 daily conditions, including sitting, walking, running, activities of daily living (ADL; eg, chores, brushing teeth), and sleeping.

Methods: One participant, (first author; 29-year-old Caucasian male) completed a 24-hour ecologically valid protocol by wearing 2 popular wrist wearable devices (Apple Watch 3 and Fitbit Charge 2). In addition, an ambulatory ECG (Vrije Universiteit Ambulatory Monitoring System) was used as the gold standard reference method, which resulted in the collection of 102,740 individual heartbeats. A single-subject design was used to keep all variables constant except for wearable devices while providing a rapid response design to provide initial assessment of wearable accuracy for allowing the research cycle to keep pace with technological advancements. Accuracy of these devices compared with the gold standard ECG was assessed using mean error, mean absolute error, and mean absolute percent error. These data were supplemented with Bland-Altman analyses and concordance class correlation to assess agreement between devices.

Results: The Apple Watch 3 and Fitbit Charge 2 were generally highly accurate across the 24-hour condition. Specifically, the Apple Watch 3 had a mean difference of -1.80 beats per minute (bpm), a mean absolute error percent of $5.86 \%$, and a mean agreement of $95 \%$ when compared with the ECG across 24 hours. The Fitbit Charge 2 had a mean difference of -3.47 bpm, a mean absolute error of $5.96 \%$, and a mean agreement of $91 \%$ when compared with the ECG across 24 hours. These findings varied by condition.

Conclusions: The Apple Watch 3 and the Fitbit Charge 2 provided acceptable heart rate accuracy $(< \pm 10 \%)$ across the 24 hour and during each activity, except for the Apple Watch 3 during the daily activities condition. Overall, these findings provide preliminary support that these devices appear to be useful for implementing ambulatory measurement of cardiac activity in research studies, especially those where the specific advantages of these methods (eg, scalability, low participant burden) are particularly suited to the population or research question.

(JMIR Mhealth Uhealth 2019;7(3):e10828) doi: 10.2196/10828 


\section{KEYWORDS}

electrocardiography; Apple Watch 3; digital health; Fitbit Charge 2; heart rate; mobile health; passive sensing; photoplethysmography; wearables

\section{Introduction}

\section{Background}

Wrist-worn smartwatches and fitness monitors or wearables have been widely adopted by consumers and are currently gaining increased attention by researchers for their potential contribution to digital measurement of health, especially in big data studies as these devices are scalable, unobtrusive, and potentially provide greater ecological validity (ie, the degree to which a research design matches naturalistic environments to generalize results to real-life settings), as compared with laboratory studies. These devices contain a multitude of sensors, often including an optical sensor that uses photoplethysmography (PPG) that allows these devices to collect pulse rate or volumetric changes in blood profusion that act as a surrogate for heart rate (HR). Although often used interchangeably, it is important to note that pulse rate and HR are 2 different physiological signals [1], with pulse rate representing the rate of change in blood pressure because of the ventricular ejection of blood, whereas HR represents the rate of heart contraction as indexed by heart electrical impulses. As such, the goal of wearable HR accuracy validation studies is to assess that device measurements, such as those between wearables and a reference method (ie, electrocardiogram; ECG), are not outside of clinically important limits of agreement (LoA), so that devices can supplement, replace, or even be used interchangeably [2].

Recently, there have been a variety of studies that have examined the accuracy of wearable PPG sensors as compared with ECG [3-9], polar chest straps [10,11], or pulse oximeters [12] across various controlled laboratory conditions, including sitting, treadmill protocols for walking and running, cycling, weight training, and sleeping. The current gold standard reference method for assessing HR is the ECG, which highlights the limitations of many studies that have utilized chest straps $[10,11]$ or pulse oximeters [12], which themselves contain a degree of error when compared with ECG. Therefore, many studies are comparing wearable HR accuracy with suboptimal comparison methods, which likely undermine findings. Below, we primarily reviewed the existing wearable HR literature that has used an ECG as the comparison method.

Previous research comparing wearables with the gold standard ECG, which uses electrodes to measure cardiac muscular contractions from electrical activity of the heart, has shown that that wearables underestimate absolute HR as compared with reference methods [3-4,6,8-11,13]. Prior research has also shown that the Apple Watch has greater accuracy than Fitbit devices [7-9]. Specifically, prior research has found that the Apple Watch has lower overall error [3,7,10], lowest mean difference [8], and higher agreement with ECG than Fitbit devices [3,9], but that wearables' accuracy depends on activity [5]. Research has shown that at rest, wearables can perform similarly to an ECG but not with moderate exercise [14]. There has been a substantial amount of research that has shown that wearable devices are more accurate during rest and low intensity exercise as compared with exercises at higher intensity [3,5,9,15-17], which may be because of the position of the device during rest [18] and less movement of the wearable device around the wrist at rest, although this is not found in all studies [7,10-11,19]. Specifically, 1 study found that there was not a significant difference in HR accuracy across baseline or vigorous activity [10], whereas a second study found that HR accuracy was highest during running - a very intense activity [19], and a third found that walking, running, and cycling were more accurate for some devices than sitting [7]. Therefore, it is possible that activity intensity may be less important to device accuracy than the degree of erratic wrist movements performed during physical activity, which tend to co-occur with more vigorous physical activity.

\section{Four Challenges Limiting Progress for Wearable Heart Rate Accuracy}

Currently, prior research has greatly improved our understanding of wearable HR accuracy, but there remain 4 challenges that limit progress in this area. First, as mentioned above, many studies lack an appropriate comparison method by opting to utilize chest straps [10,11] or pulse oximeters [12], rather than an ECG, which themselves contain a degree of error when compared with ECG. Therefore, many studies are comparing wearable HR accuracy with suboptimal comparison methods, which likely undermine findings. Second, wearable manufacturers use proprietary algorithms to translate PPG signals to HR measurements. These algorithms are likely altered with firmware updates, yet most studies fail to report firmware information. This may lead to poor reproducibility as 2 studies investigating the same device with different firmware versions might actually come to different conclusions even if all other variables are held constant. Third, almost all prior studies have utilized laboratory paradigms, rather than naturalistic settings. Recent research has called for the test of devices in the setting appropriate for intended use [20]. Although controlled laboratory settings are important for maintaining experimental control, this design involves a trade-off that often creates an artificial environment during which individual behaviors may deviate from that in naturalistic settings of lived daily experience. For example, laboratory settings tend to test specific movements within predetermined time frames, whereas consumers use wearables in naturalistic settings that often involve more variable and sporadic movements, which may not be accurately captured during laboratory paradigms. As such, the accuracy of wearables in controlled settings may deviate from accuracy during the daily living conditions of consumers. The 2 studies that were identified to have been conducted in more naturalistic settings have either occurred within a medical setting [6], which inherently does not capture the vast majority of consumer device use conditions, or only collected a maximum of 6 hours of free-living nonsleep conditions without the use of a gold standard ECG as a reference method [21]. Finally, the 
speed of wearable technological advancements often outpaces the typical research cycle [22], making it very difficult for studies to validate each new iteration of wearables. This calls for novel rapid response designs to quickly assess initial wearable HR accuracy in order for the research cycle to keep pace with technological advancements.

This study addresses each of the current limitations in wearable studies as it (1) uses a gold standard comparison method for movement within daily life-ambulatory ECG, (2) reports firmware versions, (3) increases the ecological validity of wearable HR accuracy by taking place during actual consumer device use conditions across a 24-hours period, and (4) takes place within an individual, rather than a traditional group of research participants, which creates an agile and novel rapid response design to quickly assess initial wearable HR accuracy in order for the research cycle to keep pace with technological advancements. This design also controls most between-subject variability and potential confound variables, allowing wearable devices to be the only study variable that varies; thus, providing a powerful (albeit potentially less generalizable) test of device accuracy.

\section{Study}

This study was preregistered (hypotheses and methods) with open code and data on Open Science Framework (OSF) [23]. The objective of this study was to determine the HR accuracy of 2 of the most popular wearables, the Apple Watch 3 and Fitbit Charge 2, as compared with the gold standard method for continuous recording in real-world settings-an ambulatory ECG. As mentioned above, a single-subject design was used for this initial study on the ecological validity of wearables to provide a proof-of-concept research design that will allow research cycles to keep pace with the technological advancements of wearables, while also eliminating between-subject variability. Although a single-subject design is a limitation, a recent study has highlighted the possibility that group-level findings do not apply to the individual [24] and that $\mathrm{N}$ of 1 trials are a promising approach to empirical decision making [25]. Furthermore, single-subject designs are being increasingly used [26-28], even in leading journals [29-31]. One strength of this design is that all potential confound variables can be held constant, except for the wearable devices; thus, providing a powerful test of accuracy of the devices per se.

This study hypothesized that (1) the Apple Watch 3 would be more accurate at measuring HR than the Fitbit Charge 2 when compared with an ambulatory ECG across all conditions, (2) both wearables would underestimate HR across all conditions, and (3) device measurement of HR would become increasingly inaccurate as activity intensity increased.

\section{Methods}

\section{Recruitment}

We investigated the accuracy of wearable HR from 2 popular devices in a single healthy human (first author) who completed a 24-hour protocol. The participant (29-years-old Caucasian male; body mass index=21.1; Fitzpatrick skin tone measure $=2$; right wrist $(\mathrm{cm})=7.0$; left wrist $(\mathrm{cm})=6.5$; right hand dominant $)$ conceptualized and initiated this study, with the purpose of having the data published. Therefore, approval from the University of Oregon ethics committee was unnecessary and not obtained. The first author gave consent for collecting and using the data for study purposes.

\section{Study Protocol}

Participant's psychophysiology recordings began at 18:28 on day 1 and briefly stopped at 17:10 on day 2 before the run condition. Recording resumed at 17:37 for the run condition and stopped at 18:50 on day 2. Age, gender, height, and weight were used to set up both wearable devices.

\section{Conditions}

A total of 5 daily conditions were recorded throughout the 24-hour study using a digital notebook (Google Sheets) to record activity times, resulting in 84 start and stop marker times. These included sitting, which included any seated activity; walking; running (this occurred on a treadmill to allow for a stable ambulatory ECG signal); activities of daily living (ADL), which included activities such as cleaning, brushing teeth, and cooking; and sleeping. Although prior research has excluded HR data during activity transitions, these were not excluded in this study to preserve ecological validity of device usage in real-world conditions. Therefore, although transition periods generally yield higher device error, we wanted to capture this variability as part of device accuracy in this study.

\section{Gold Standard Reference Method}

ECG data were acquired using a standard 3-lead ambulatory ECG (Vrije Universiteit Ambulatory Monitoring System) [32,33]. ECG sampling frequencies were $1000 \mathrm{~Hz}$, and HR was exported in 1-min epochs, from 00 seconds to 59 seconds.

\section{Wearable Devices}

\section{Apple Watch 3}

The Apple Watch Series 3 (2017 version, Apple Inc, California, USA, v. 4.2.3) $42 \mathrm{~mm}$ was worn on the right wrist. According to Apple, the Apple Watch 3 samples HR approximately every 10 min or continuously during workouts using PPG with either a green light emitting diode or infrared light and photodiode sensors. In other words, during this study, the Apple Watch 3 collected HR data as would occur in real-world conditions, continuously for walking and running and approximately every 10 min during all other activities. The Apple Watch 3 was synced with the Apple Health app on the iPhone and then exported in XML format for analysis. The Apple Health Analysis GitHub repository [34] was used to convert the XML file to a data frame in $\mathrm{R}$ Studio to access per min data for analysis. When more than 1 heart rate measurement was collected each min during continuous HR recording for walking and running activities, the average of these measurements was used in line with prior wearable research [7].

\section{Fitbit Charge 2}

The Fitbit Charge 2 (2017 version, Fitbit Inc, California, USA, v. 22.55.2) was worn on the left wrist. According to Fitbit, the PurePulse PPG technology utilizes green LED light to continuously index HR. The Fitbit GitHub repository [35] was 
used to interact with the Fitbit app programming interface to access per minute data for analysis.

\section{Error}

To assess error, we used mean error (ME), mean absolute error (MAE), and mean absolute percent error (MAPE). In line with prior wearable research $[3,11,23,36,37]$ as well as recommendations from the Association for the Advancement of Medical Instrumentation, the Consumer Technology Association [38] and the American National Standards Institute [39], we defined an acceptable error rate for a physical monitoring device to be $\pm 10 \%$, as this is considered an accurate threshold for medical ECG monitors. We recognize that this is more lenient than some prior health sciences research on wearable HR accuracy [7] and pedometer step counting accuracy $[40,41]$ that have defined an acceptable error rate to be $\pm 5 \%$. In line with recent recommendations [37,38], we used MAPE to determine acceptable error rate. Outliers were not removed as this would interfere with determining device accuracy during consumer use conditions.

\section{Statistical Analysis}

All analyses were performed in R (version 3.4.3) using R Studio (version 1.1.383). Scripts can be found on GitHub [34,35] and OSF [23]. Data can be found on OSF. Analyses were performed using the beats per minute (bpm) separately for each wearable device as compared with the gold standard ECG data for HR calculated as bpm.

\section{Mean Error}

The ME was calculated as the difference between the device measurement and the gold standard measurement.

\section{Mean Absolute Error}

The MAE was calculated as the average absolute distance between the device measurement and the gold standard measurement.

\section{Mean Absolute Percent Error}

The MAPE relative to the ECG was calculated for each wearable device by averaging the individual absolute percent errors.

\section{Bland-Altman Analysis}

Bland-Altman analysis and 95\% LoA were calculated using the blandr [42] and BlandAltmanLeh $\mathrm{R}$ packages [43]. This is the recommended method to determine agreement between medical instruments $[2,44]$, rather than other methods of agreement, because it is unlikely that devices will have an exact agreement, and therefore, the importance lies in how close pairs of observations are, as small differences between devices are unlikely to impact patient decisions [45].

\section{Concordance Class Correlation}

Finally, although not one of the analyses that was preregistered, we also ran concordance class correlation (CCC) analyses between the ECG and each wearable device separately across all conditions using the DescTools R Package [46] to assist in Bland-Altman plot interpretation. In line with prior wearable research [8], the strength of agreement was interpreted based on the following, weak $(\mathrm{CCC}<.5)$, moderate $(\mathrm{CCC}=.5-.7)$, and strong $(\mathrm{CCC}>.7)$.

\section{Results}

\section{Descriptives}

The ECG collected 1424 HR observations, the Apple Watch 3 collected 394 HR observations (only collects measurements every 10 min, except during walking and running), and the Fitbit Charge 2 collected 1425 observations, resulting in a total of 3243 HR observations across devices (see Figure 1). See Table 1 for number of observations and HR descriptive statistics for each condition. See Figure 2 for descriptives of HR trajectories across the 24 hours with activity type (note that the bottom figure has less resolution as the Apple Watch 3 collected HR every $10 \mathrm{~min}$, except for walking and running conditions). 
Figure 1. Rainbow plot of heart rate observations for electrocardiogram (ECG), Fitbit Charge 2, and Apple Watch 3. bpm: beats per minute.

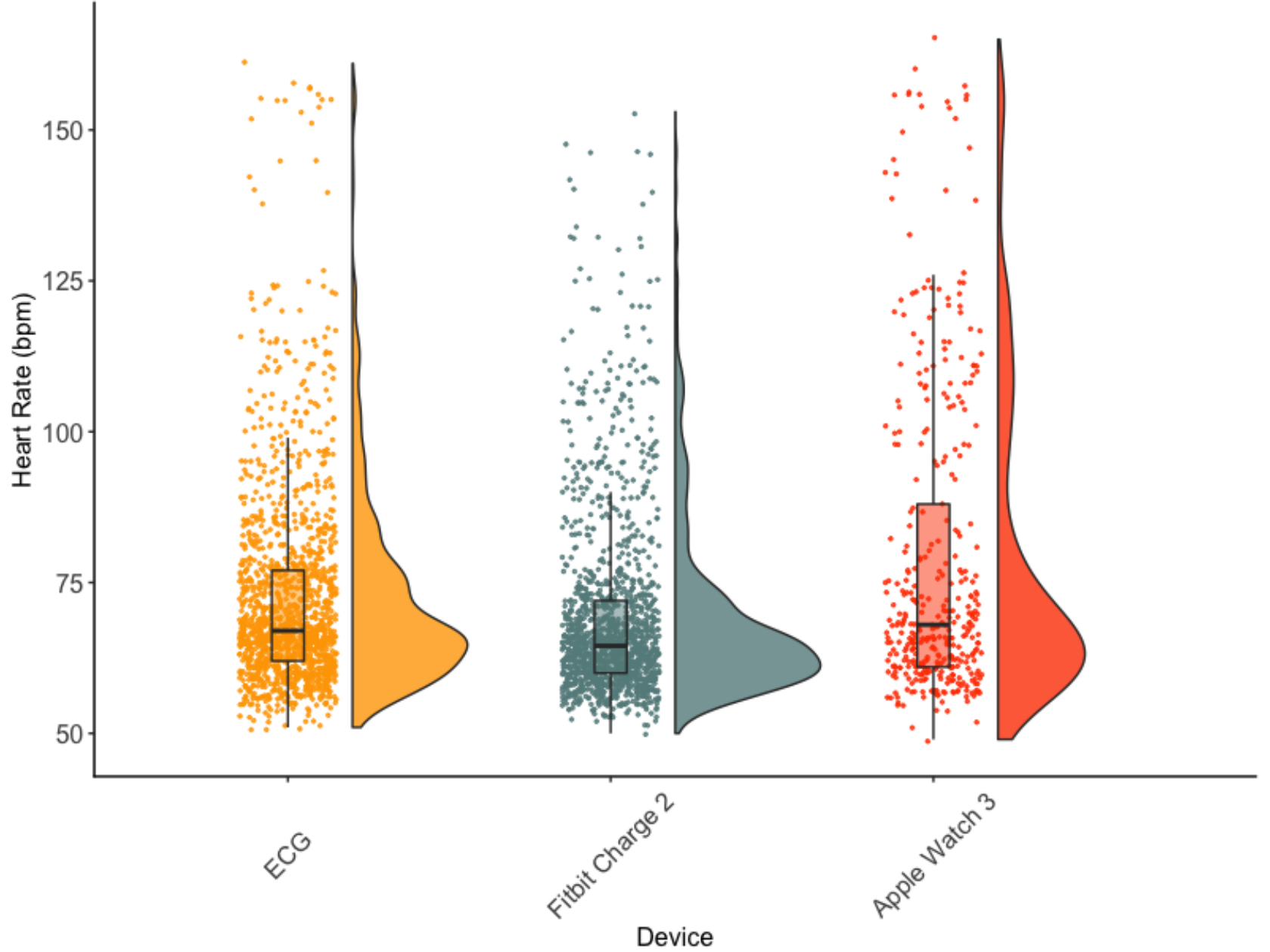


Table 1. Heart rate descriptive statistics by condition.

\begin{tabular}{|c|c|c|c|}
\hline Activity and device & Observations, $\mathrm{n}$ & Heart rate, mean (SD) & Heart rate range \\
\hline \multicolumn{4}{|l|}{24 hours } \\
\hline $\mathrm{ECG}^{\mathrm{a}}$ & 1424 & $72.65(16.92)$ & $51-161$ \\
\hline Apple Watch 3 & 394 & $78.78(25.74)$ & $49-165$ \\
\hline Fitbit Charge 2 & 1446 & $69.10(15.10)$ & $50-153$ \\
\hline \multicolumn{4}{|l|}{ Sitting } \\
\hline ECG & 535 & $70.41(7.24)$ & $55-97$ \\
\hline Apple Watch 3 & 144 & $67.91(7.69)$ & $54-98$ \\
\hline Fitbit Charge 2 & 535 & $65.72(5.51)$ & $55-91$ \\
\hline \multicolumn{4}{|l|}{ Walking } \\
\hline ECG & 100 & $102.32(16.87)$ & $61-127$ \\
\hline Apple Watch 3 & 79 & $106.06(15.03)$ & $55-139$ \\
\hline Fitbit Charge 2 & 100 & $95.47(17.88)$ & $54-132$ \\
\hline \multicolumn{4}{|l|}{ Running } \\
\hline ECG & 22 & $147.82(13.13)$ & $104-161$ \\
\hline Apple Watch 3 & 22 & $149.59(10.24)$ & $120-165$ \\
\hline Fitbit Charge 2 & 22 & $133.09(12.72)$ & $95-153$ \\
\hline \multicolumn{4}{|c|}{ Activities of daily living } \\
\hline ECG & 216 & $84.16(11.28)$ & $58-115$ \\
\hline Apple Watch 3 & 34 & $74.94(14.53)$ & $52-125$ \\
\hline Fitbit Charge 2 & 214 & 80.38 (13.08) & $56-121$ \\
\hline \multicolumn{4}{|l|}{ Sleeping } \\
\hline ECG & 551 & $61.93(4.94)$ & $51-78$ \\
\hline Apple Watch 3 & 110 & $60.60(4.06)$ & $49-73$ \\
\hline Fitbit Charge 2 & 551 & $60.82(4.40)$ & $50-74$ \\
\hline
\end{tabular}

${ }^{\mathrm{a} E C G: ~ e l e c t r o c a r d i o g r a m . ~}$ 
Figure 2. Fitbit Charge 2 (top) and Apple Watch 3 (bottom) compared to the electrocardiogram (ECG) across 24-hours. bpm: beats per minute.
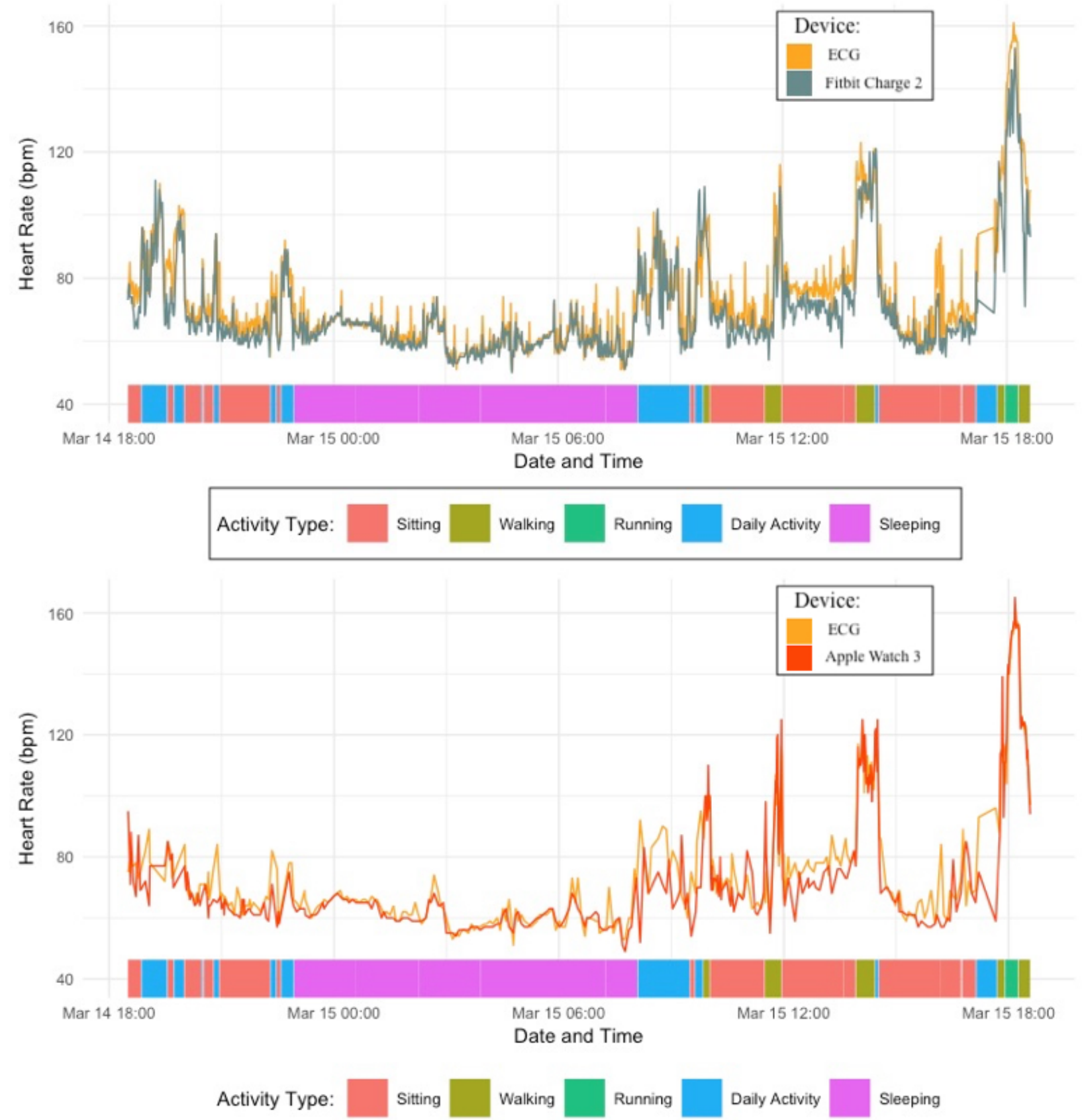

\section{Percent Error}

Overall, across the 24-hour recording, the Apple Watch 3 had a MAPE of $5.86 \%$, whereas the Fitbit Charge 2 had MAPE of $5.96 \%$. During sitting conditions, the Apple Watch 3 had a MAPE of $7.21 \%$, whereas the Fitbit Charge 2 had a MAPE of $6.93 \%$. During walking conditions, the Apple Watch 3 had a MAPE of $4.64 \%$, whereas the Fitbit Charge 2 had a MAPE of
9.21\%. During the running condition, the Apple Watch 3 had a MAPE of 3.01\%, whereas the Fitbit Charge 2 had a MAPE of $9.88 \%$. During ADL, the Apple Watch 3 had a MAPE of $13.70 \%$, whereas the Fitbit Charge 2 had a MAPE of $8.29 \%$. Finally, during the sleep condition, the Apple Watch 3 had a MAPE of $3.12 \%$, whereas the Fitbit Charge 2 had a MAPE of $3.36 \%$ (see Table 2 for percent error statistics and Figure 3 for MAPE by device across activities). 
Table 2. Device error statistics and Bland-Altman analyses.

\begin{tabular}{|c|c|c|c|c|c|}
\hline \multirow[t]{2}{*}{ Activity and device } & \multicolumn{3}{|l|}{ Device error } & \multicolumn{2}{|c|}{ Bland-Altman analysis } \\
\hline & Mean absolute error & Mean absolute percent error ${ }^{\mathrm{a}}(\%)$ & Mean error (SD) & Lower LoA ${ }^{b}$ & Upper LoA \\
\hline \multicolumn{6}{|l|}{24 hours } \\
\hline Apple Watch 3 & 4.72 & 5.86 & $-1.80(7.40)$ & -16.31 & 12.71 \\
\hline Fitbit Charge 2 & 4.71 & 5.96 & $-3.47(6.17)$ & -15.55 & 8.62 \\
\hline \multicolumn{6}{|l|}{ Sitting } \\
\hline Apple Watch 3 & 5.24 & 7.21 & $-2.47(7.39)$ & -16.94 & 12.01 \\
\hline Fitbit Charge 2 & 5.93 & 6.93 & $-4.69(4.90)$ & -14.29 & 4.91 \\
\hline \multicolumn{6}{|l|}{ Walking } \\
\hline Apple Watch 3 & 4.77 & 4.64 & $0.11(7.29)$ & -14.18 & 14.41 \\
\hline Fitbit Charge 2 & 9.55 & 9.21 & $-6.85(11.05)$ & -28.51 & 14.81 \\
\hline \multicolumn{6}{|l|}{ Running } \\
\hline Apple Watch 3 & 4.05 & 3.01 & $1.77(5.90)$ & -9.78 & 13.33 \\
\hline Fitbit Charge 2 & 14.73 & 9.88 & $-14.73(7.67)$ & -29.77 & 0.31 \\
\hline \multicolumn{6}{|c|}{ Activities of daily living } \\
\hline Apple Watch 3 & 11.74 & 13.70 & $-8.50(12.90)$ & -33.78 & 16.78 \\
\hline Fitbit Charge 2 & 7.05 & 8.29 & $-3.73(8.24)$ & -19.88 & 12.41 \\
\hline \multicolumn{6}{|l|}{ Sleeping } \\
\hline Apple Watch 3 & 1.96 & 3.12 & $-0.95(2.78)$ & -6.39 & 4.50 \\
\hline Fitbit Charge 2 & 2.15 & 3.36 & $-1.11(3.20)$ & -7.28 & 5.17 \\
\hline
\end{tabular}

${ }^{\mathrm{a}}$ Validity was established as devices having a MAPE value $<10 \%$.

${ }^{\mathrm{a}} \mathrm{LoA}$ : limits of agreement.

Figure 3. Mean absolute percent error (MAPE) by device across types of activities. Note: Horizontal line represents threshold for validity. 20 -

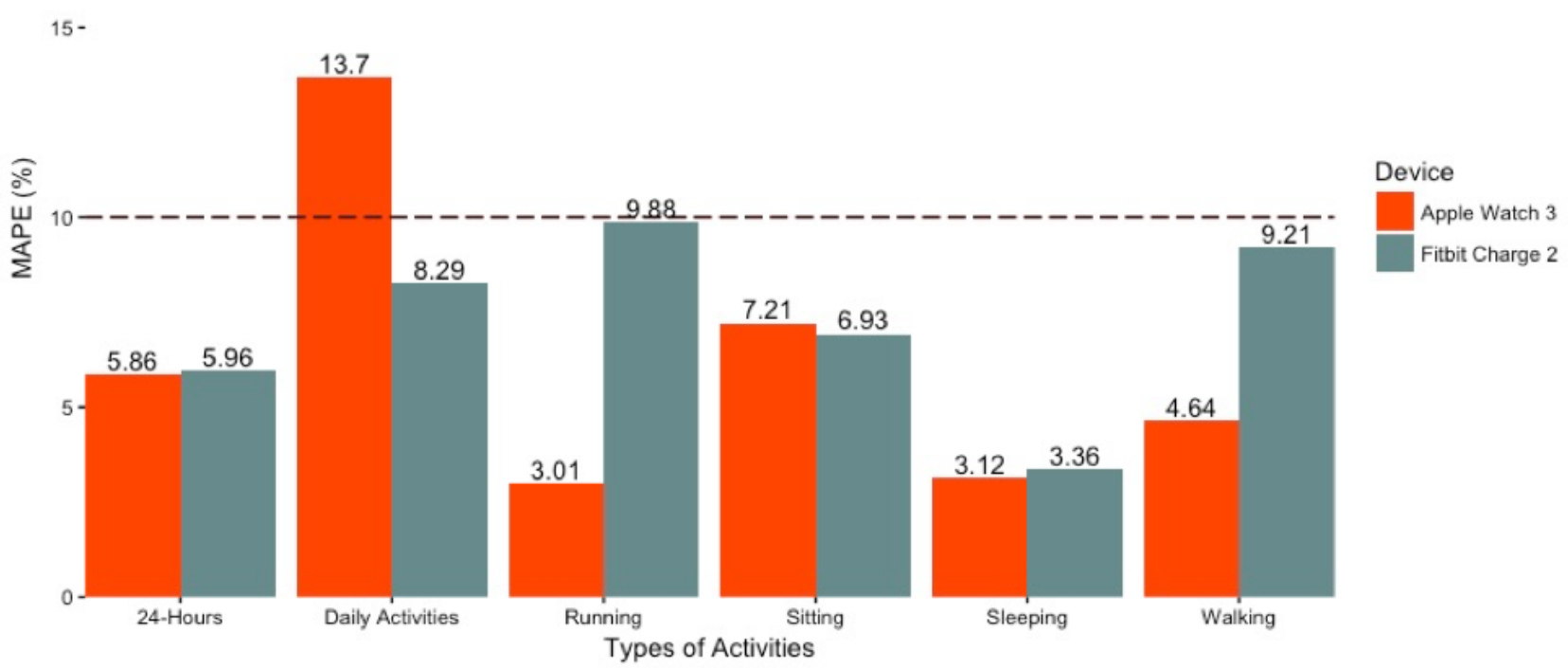




\section{Bland-Altman Analysis and 95 Percent Limits of Agreement}

Overall, across the 24-hour recording (see Figure 4), the Apple Watch 3 had an ME of $-1.80 \mathrm{bpm}$ (lower LoA-upper LoA: -16.31 to $12.71 \mathrm{bpm}$ ) and an MAE of 4.72, whereas the Fitbit Charge 2 had an ME of -3.47 bpm (lower LoA-upper LoA: -15.54 to $8.62 \mathrm{bpm}$ ) and an MAE of 4.71. Visual inspection of the Bland-Altman plots revealed a tendency for the Apple Watch 3 to both over- and underestimate HR values when observations were between 70 bpm to120 bpm, whereas the Fitbit Charge 2 had a tendency to underestimate HR values, particularly once HR values exceeded approximately 80 bpm (see Table 3 for Bland-Altman statistics).

During sitting conditions, the Apple Watch 3 had an ME of -2.47 bpm (lower LoA-upper LoA: -16.94 to $12.01 \mathrm{bpm}$ ) and an MAE of 5.24, whereas the Fitbit Charge 2 had an ME of -4.69 bpm (lower LoA-upper LoA; -14.29 to $4.91 \mathrm{bpm}$ ) and an MAE of 5.93. During walking conditions, the Apple Watch
$3 \mathrm{had}$ an ME of $0.11 \mathrm{bpm}$ (lower LoA-upper LoA: -14.18 to $14.41 \mathrm{bpm}$ ) and an MAE of 4.77, whereas the Fitbit Charge 2 had an ME of $-6.85 \mathrm{bpm}$ (lower LoA-upper LoA: -28.51 to $14.81 \mathrm{bpm}$ ) and an MAE of 9.55. During the running condition, the Apple Watch 3 had an ME of 1.77 bpm (lower LoA-upper LoA: 9.78 to $13.33 \mathrm{bpm}$ ) and an MAE of 4.05, whereas the Fitbit Charge 2 had an ME of -14.73 bpm (lower LoA-upper LoA: -29.77 to $0.31 \mathrm{bpm}$ ) and an MAE of 14.73. During ADL, the Apple Watch 3 had an ME of $-8.50 \mathrm{bpm}$ (lower LoA-upper LoA: -33.78 to $16.78 \mathrm{bpm}$ ) and an MAE of 11.74, whereas the Fitbit Charge 2 had an ME of -3.73 bpm (lower LoA-upper LoA: -19.88 to $12.41 \mathrm{bpm}$ ) and an MAE of 7.05. Finally, during the sleep condition, the Apple Watch 3 had an ME of -0.95 bpm (lower LoA-upper LoA: -6.39 to $4.50 \mathrm{bpm}$ ) and an MAE of 1.96, whereas the Fitbit Charge 2 had an ME of $-1.11 \mathrm{bpm}$ (lower LoA-upper LoA: -7.28 to $5.17 \mathrm{bpm}$ ) and an MAE of 2.15 (see Table 3 for device error and Bland-Altman statistics and Figures 5-9 for Bland-Altman plots by activity type).

Figure 4. Bland-Altman plot and density plots across 24-hours of the Apple Watch 3 (left) with 394 heart rate observations and Fitbit Charge 2 (right) with 1425 heart rate observations.
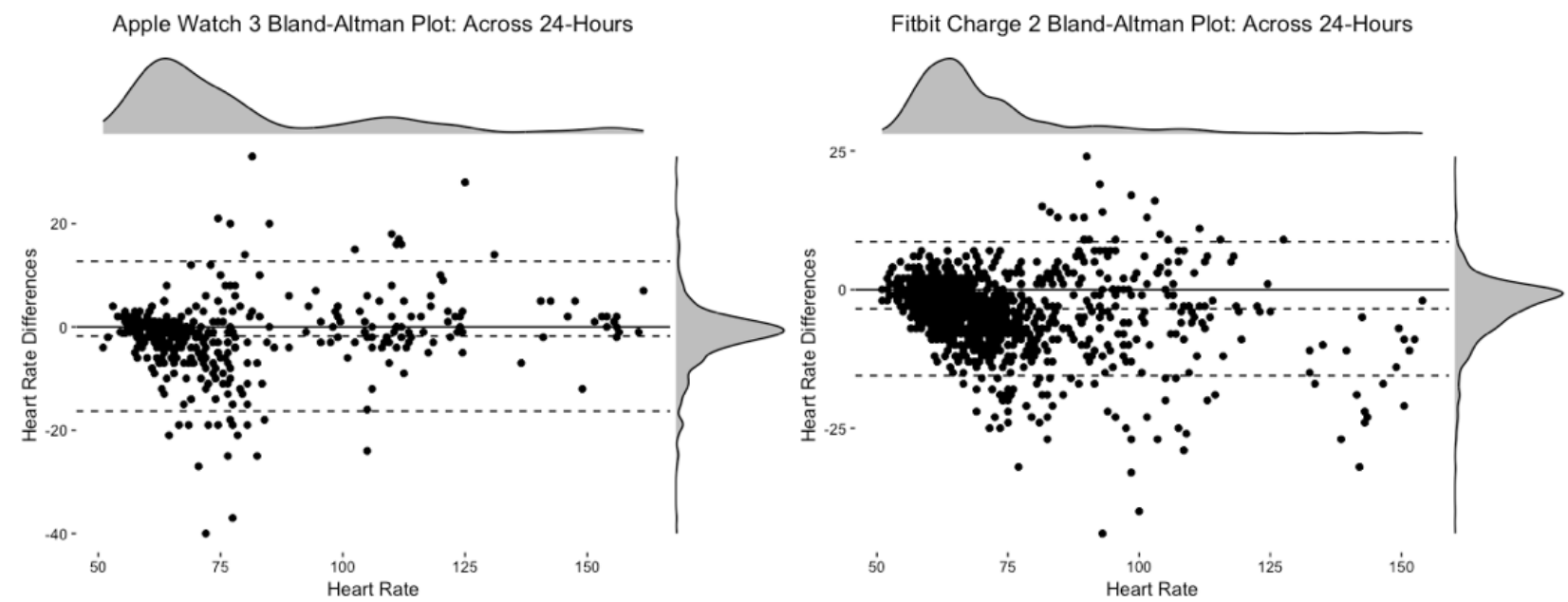
Table 3. Device error statistics and Bland-Altman analyses.

\begin{tabular}{|c|c|c|c|c|}
\hline \multirow[t]{2}{*}{ Activity and device } & \multicolumn{2}{|l|}{ Device error } & \multicolumn{2}{|c|}{ Bland-Altman analysis } \\
\hline & Mean absolute error & Mean error (SD) & Lower LoA ${ }^{\mathrm{a}}$ & Upper LoA \\
\hline \multicolumn{5}{|l|}{24 hours } \\
\hline Apple Watch 3 & 4.72 & $-1.80(7.40)$ & -16.31 & 12.71 \\
\hline Fitbit Charge 2 & 4.71 & $-3.47(6.17)$ & -15.55 & 8.62 \\
\hline \multicolumn{5}{|l|}{ Sitting } \\
\hline Apple Watch 3 & 5.24 & $-2.47(7.39)$ & -16.94 & 12.01 \\
\hline Fitbit Charge 2 & 5.93 & $-4.69(4.90)$ & -14.29 & 4.91 \\
\hline \multicolumn{5}{|l|}{ Walking } \\
\hline Apple Watch 3 & 4.77 & $0.11(7.29)$ & -14.18 & 14.41 \\
\hline Fitbit Charge 2 & 9.55 & $-6.85(11.05)$ & -28.51 & 14.81 \\
\hline \multicolumn{5}{|l|}{ Running } \\
\hline Apple Watch 3 & 4.05 & $1.77(5.90)$ & -9.78 & 13.33 \\
\hline Fitbit Charge 2 & 14.73 & $-14.73(7.67)$ & -29.77 & 0.31 \\
\hline \multicolumn{5}{|c|}{ Activities of daily living } \\
\hline Apple Watch 3 & 11.74 & $-8.50(12.90)$ & -33.78 & 16.78 \\
\hline Fitbit Charge 2 & 7.05 & $-3.73(8.24)$ & -19.88 & 12.41 \\
\hline \multicolumn{5}{|l|}{ Sleeping } \\
\hline Apple Watch 3 & 1.96 & $-0.95(2.78)$ & -6.39 & 4.50 \\
\hline Fitbit Charge 2 & 2.15 & $-1.11(3.20)$ & -7.28 & 5.17 \\
\hline
\end{tabular}

${ }^{\mathrm{a}} \mathrm{LoA}$ : limit of agreement.

Figure 5. Bland-Altman plots by daily activity. Left: Apple Watch 3 during sitting; right: Fitbit Charge 2 during sitting.

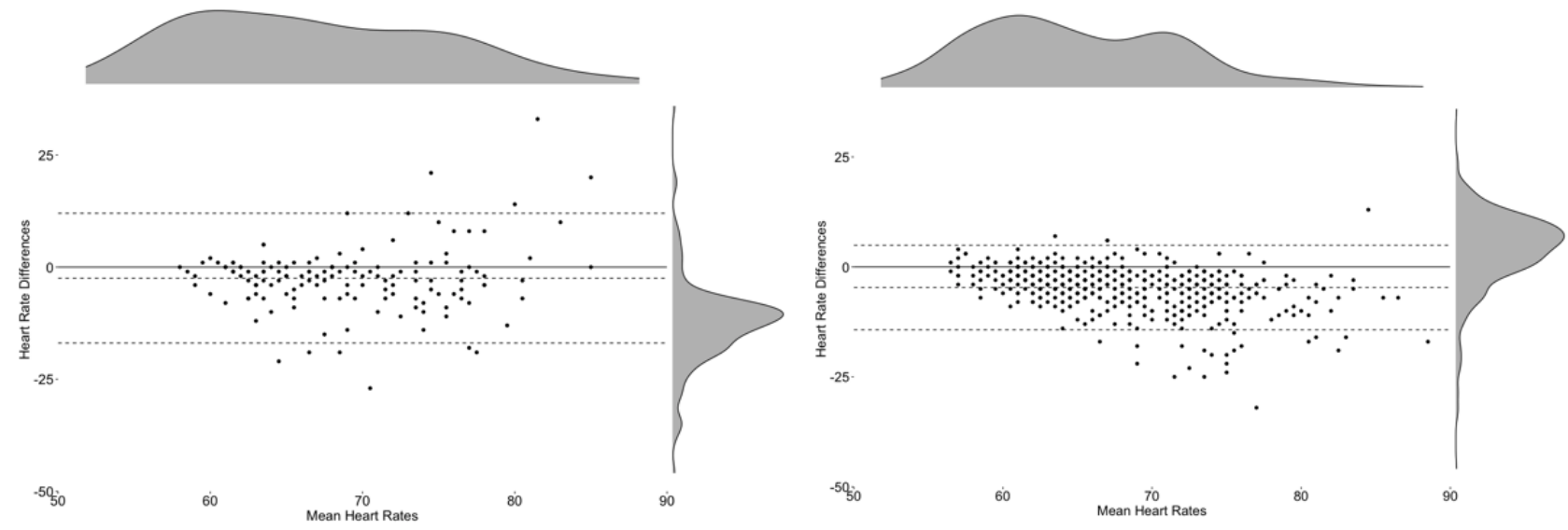


Figure 6. Bland-Altman plots by daily activity. Left: Apple Watch 3 during walking; right: Fitbit Charge 2 during walking.

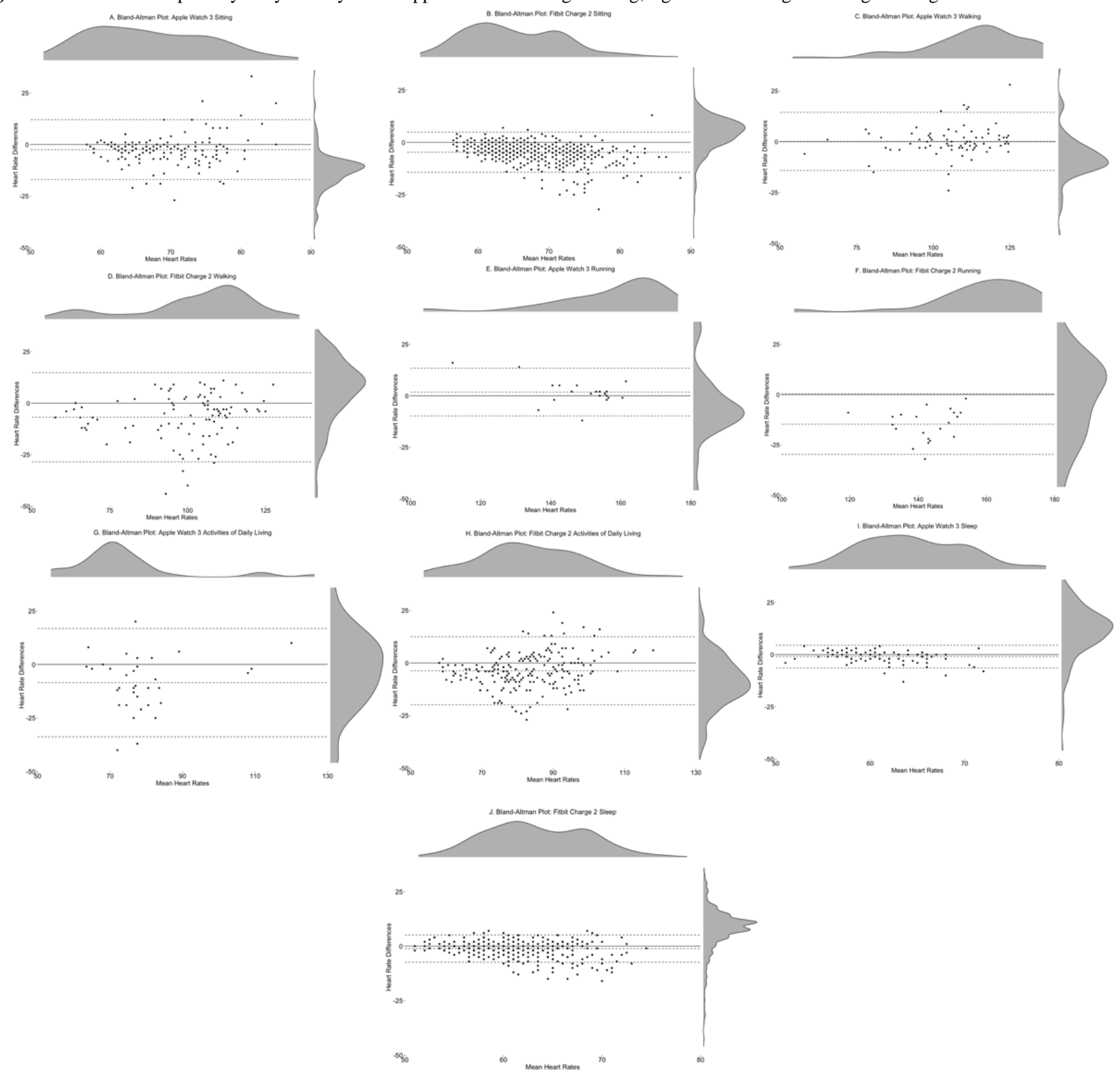

Figure 7. Bland-Altman plots by daily activity. Left: Apple Watch 3 during running; right: Fitbit Charge 2 during running.

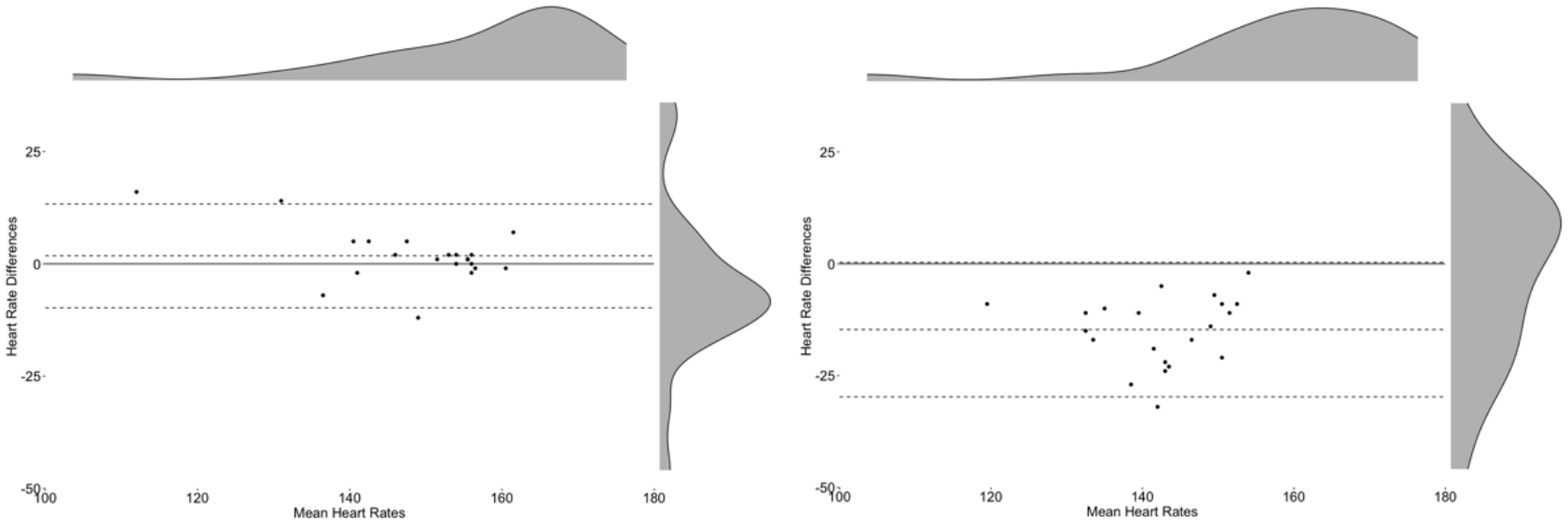


Figure 8. Bland-Altman plots by daily activity. Left: Apple Watch 3 during activities of daily living; right: Fitbit Charge 2 during activities of daily living.
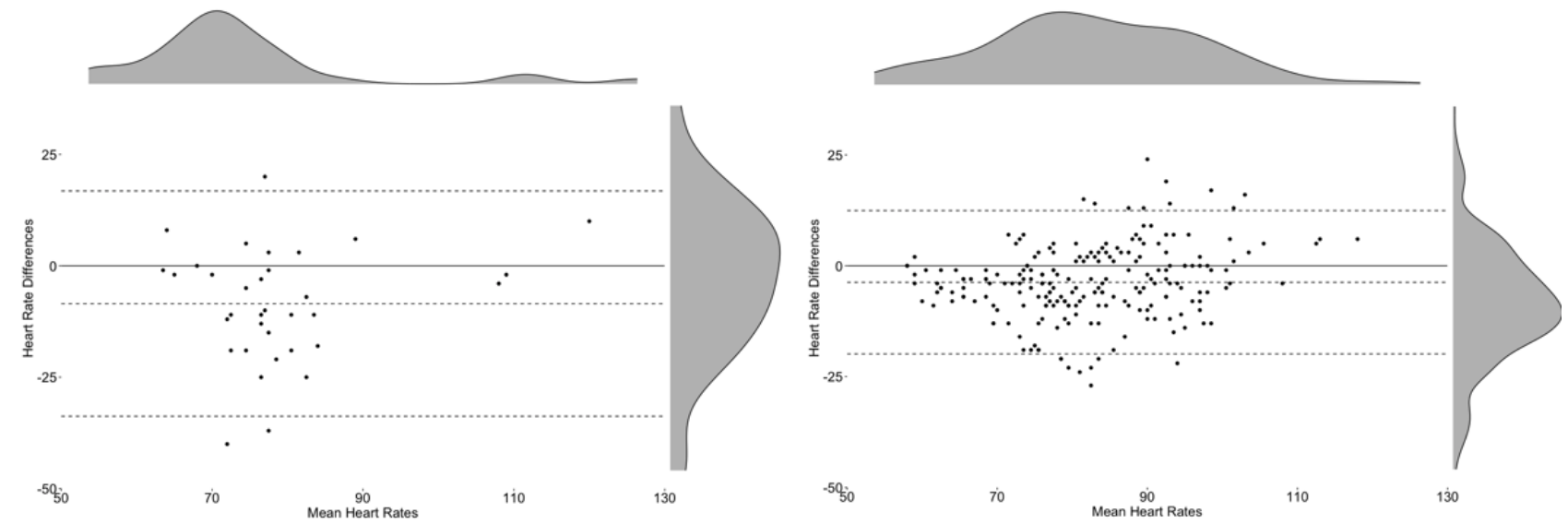

Figure 9. Bland-Altman plots by daily activity. Left: Apple Watch 3 during sleep; right: Fitbit Charge 2 during sleep.
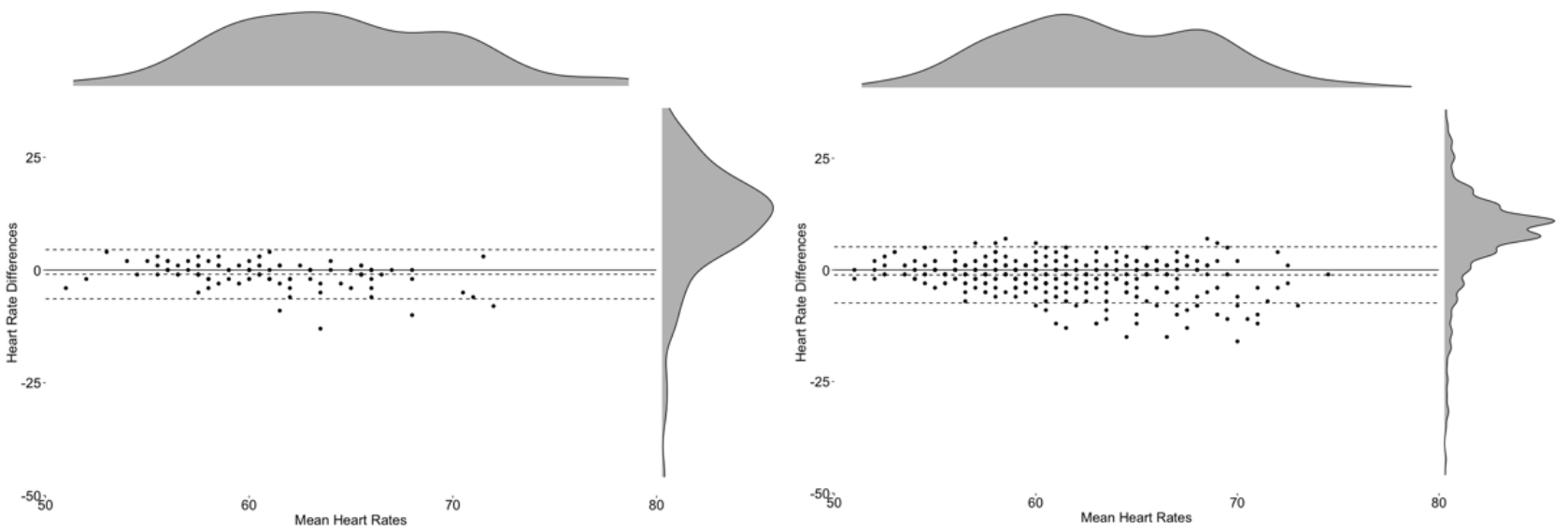

\section{Concordance Class Correlation}

Overall, across the 24-hour recording, the Apple Watch 3 (CCC $=.955$, 95\% CI 0.945-0.963) and the Fitbit Charge 2 (CCC $=.906,95 \%$ CI 0.896-0.914) had strong agreement with the reference method. During sitting conditions, the Apple Watch 3 (CCC $=.453,95 \%$ CI 0.321-0.567) had weak agreement and the Fitbit Charge 2 (CCC $=.561,95 \%$ CI $0.515-0.603)$ had moderate agreement with the reference method. During all walking activities, the Apple Watch $3(\mathrm{CCC}=.871,95 \% \mathrm{CI}$ 0.807-0.915) and the Fitbit Charge 2 ( $\mathrm{CCC}=.740,95 \% \mathrm{CI}$ 0.645-0.812) had strong agreement with the reference method. During the running condition, the Apple Watch 3 (CCC $=.864$, 95\% CI 0.731-0.934) had strong agreement with the reference method, whereas the Fitbit Charge $2(\mathrm{CCC}=.490,95 \% \mathrm{CI}$ 0.268-0.663) had weak agreement with the reference method. During the ADL condition, the Apple Watch 3 (CCC $=.460$, 95\% CI 0.204-0.656) had weak agreement with the reference method, whereas the Fitbit Charge 2 (CCC $=.739$, 95\% CI 0.676-0.791) had strong agreement with the reference method. Finally, during the sleep condition, the Apple Watch 3 (CCC $=.791,95 \%$ CI $0.715-0.849)$ and the Fitbit Charge 2 (CCC $=.745,95 \%$ CI $0.707-0.779$ ) had strong agreement with the reference method.

\section{Discussion}

\section{Principal Findings}

This study provided the first continuous and ecologically valid assessment of the accuracy of the Apple Watch 3 and the Fitbit Charge 2 as they were devised to be used by consumers (ie, during ecologically valid daily activities) during a 24-hour paradigm of consumer device use conditions.

In line with previous controlled laboratory research $[4,5,7-9,11,12]$, our findings indicated that both wearable devices provided acceptable overall aggregated accuracy $(<10 \%$ MAPE) across the 24-hour recording period as well as during each type of activity, except for the Apple Watch 3 during ADL. In addition, in line with previous research, both the Apple Watch 3 and the Fitbit Charge 2 slightly underestimated HR across the 24-hour study as compared with ECG and other reference methods [3,4,6,8-11], although this underreporting of absolute $\mathrm{HR}$ is unlikely to be problematic in most contexts as this was less than $5 \mathrm{bpm}$. Although these wearables slightly underestimated HR when values were aggregated by activity, there were a number of individual observations that were inaccurate by significantly large margins, which would be problematic in some contexts (eg, medical settings). This has potential implications for liability of device usage in medical settings [47], indicating that although overall summary statistics 
may be very accurate for research purposes, any single observation in real time may have a large degree of error, which could be significant for moment-to-moment observations in medical settings. In addition, we found it surprising that the Apple Watch 3 had such a high MAPE (13.70\%) during ADL as compared with the Fitbit Charge 2 MAPE (8.29\%). This difference was likely because of the fact that the Apple Watch 3 was worn on the dominant hand, which may have made more erratic movements than the Fitbit Charge 2 on the nondominant hand during ADL. In other words, this may have potentially moved the position of the wearable more frequently on the dominant hand, making it more difficult for the PPG sensor to assess and accurately measure HR, as has been found in prior studies [18].

Overall, the Apple Watch 3 had acceptable error across the entire 24-hour period as well as all activities except when the error rate rose above the $\pm 10 \%$ threshold for the Apple Watch 3 during ADL (13.70\%), while the Fitbit Charge 2 had acceptable error across the entire 24-hour period as well as all activities, although its error got close to the $\pm 10 \%$ threshold during walking $(9.21 \%)$ and running $(9.88 \%)$. In addition, both devices slightly underestimated heart rate. Finally, as movement became more erratic during certain conditions and as HR increased, the devices became less accurate.

\section{Strengths and Limitations}

This study had a number of strengths that addressed 4 current limitations in wearable studies: this study (1) used a gold standard comparison method for movement within daily life-ambulatory ECG, (2) reported firmware numbers, (3) increased the ecological validity of wearable HR accuracy by taking place during actual consumer device use conditions across a 24-hour period, and (4) took place within an individual, rather than a traditional group of research participants, which creates a novel rapid response design to quickly assess initial wearable HR accuracy in order for the research cycle to keep pace with technological advancements while also controlling for between-subject variability and most potential confounding variables, which allowed for the wearable devices to be the only study variable that varied, thus, providing a powerful test of device accuracy. Furthermore, prior research has shown that there is a 24-hour circadian rhythm to HR [48] and that this can be particularly important as adverse cardiovascular events such as heart attacks, stroke, and cardiac deaths tend to occur in the late mornings [49]. The approach of this study also captured the 24-hour circadian rhythm HR from 3 different devices during real-life conditions, which indicates that these devices can detect changes in HR across the day.

In addition to these strengths, there were also a number of limitations. First, the single-subject design limited various participant demographic factors, such as body mass index, skin tone, and wrist circumference, which have been shown to correlate with HR error rate [7,16]. Future studies should attempt to replicate these results across multiple individuals with diverse body mass index, wrist circumference, skin tone, fitness level, and stress level. Another limitation in this study was that the Fitbit Charge 2 and Apple Watch 3 collected HR measurements at different frequencies. Specifically, the Fitbit Charge 2 recorded an HR measurement each minute, whereas the Apple Watch 3 collected continuous HR measurements during walking and running tasks (the average of these measurements was used for each minute in line with prior research [7]) and every 10 min for all other activities. This discrepancy in device sampling rates combined with proprietary underlying algorithms for the way per minute HR is calculated for each wearable device might help account for the lower reported accuracy of the Apple Watch 3 during the ADL condition. In addition, the single-subject design combined with the Apple Watch 3 sampling rate of approximately every $10 \mathrm{~min}$ led to a small number of observations for some conditions. Although continuous recording was not activated on the Apple Watch 3 to approximate real-world usage conditions, future studies should aim to collect larger numbers of subjects to increase the observations for each condition and potentially activate continuous recording on this device. Similarly, although this study had the strength of providing the first 24-hour continuous and ecologically valid assessment of wearable accuracy in real-world conditions, this was also a limitation as this design inherently could not take place within more controlled laboratory settings that used a stationary ECG, rather than an ambulatory ECG that may introduce some additional error. In fact, the running condition had to take place on a treadmill to keep the ECG device stable enough to prevent excessive artifacts. Another limitation of this study is that although that overall error rates of both devices were low, there were some individual observations that were inaccurate by significantly large margins. This indicates that although overall summary statistics for conditions may be very accurate, any single observation in real time may have a large degree of error. Researchers should keep this in mind when using wearable devices in research settings, and this finding emphasizes the importance of data cleaning. Implementing these devices in research settings would likely benefit from automated outlier detection and deletion techniques as would the underlying scoring algorithms. Finally, this study did not counterbalance wrist placement of the wearables to rule out potential influences of wrist circumference, musculature, or movement on the accuracy of HR readings. The subject was right-handed, and therefore, the lower accuracy of the Apple Watch 3 as compared with the Fitbit Charge 2 during the ADL condition may have been because of more erratic wrist motions that accompany many activities in this condition as prior research has indicated that the lack of smooth wrist movements introduces larger HR measurement error [10]. Future studies should provide both between-subjects analyses and within-subjects analyses with devices on both wrists to assess the accuracy of wearables, as hand dominance may influence accuracy.

\section{Conclusions}

This study provided the first continuous and ecologically valid assessment of the accuracy of the Apple Watch 3 and the Fitbit Charge 2 HR measurements as they were devised to be used by consumers out in the real world during a 24-hour paradigm of actual consumer device use conditions. Overall, both the Apple Watch 3 and Fitbit Charge 2 had acceptable HR accuracy when aggregated overall across the 24-hour period and during each condition, except for the Apple Watch 3 during the ADL 
condition. In addition, both the Apple Watch 3 and Fitbit Charge 2 slightly underestimated HR. Furthermore, both erratic wrist movements and higher HR were associated with lower device accuracy. It is important to note that although overall HR accuracy statistics for most conditions were acceptable, there were a number of individual observations that varied widely from the gold standard ECG, which indicates that any single measurement viewed in real time cannot be interpreted as an accurate measurement that has implications for medical liability of device usage [47]. Overall, wearable devices likely will not be replacing the gold standard ECG in a medical setting anytime soon, but both the Apple Watch 3 and the Fitbit Charge 2 can be used to supplement these gold standard methods in research and clinical applications. They may be particularly useful in big data studies as these devices had acceptable error rate in almost all activities while being relatively cheap, mobile, unobtrusive, and scalable as compared with gold standard medical equipment.

\section{Conflicts of Interest}

None declared.

\section{References}

1. Lang M. Beyond Fitbit: a critical appraisal of optical heart rate monitoring wearables and apps, their current limitations and legal implications. Alb L J Sci Tech 2017;28(39):39-72 [FREE Full text]

2. Bland JM, Altman DG. Measuring agreement in method comparison studies. Stat Methods Med Res 1999;8(2):135-160 [FREE Full text] [Medline: 10501650]

3. Boudreaux BD, Hebert EP, Hollander DB, Williams BM, Cormier CL, Naquin MR, et al. Validity of wearable activity monitors during cycling and resistance exercise. Med Sci Sports Exerc 2018 Dec;50(3):624-633. [doi: 10.1249/MSS.0000000000001471] [Medline: 29189666]

4. de Zambotti M, Baker FC, Willoughby AR, Godino JG, Wing D, Patrick K, et al. Measures of sleep and cardiac functioning during sleep using a multi-sensory commercially-available wristband in adolescents. Physiol Behav 2016 May 01;158:143-149 [FREE Full text] [doi: 10.1016/j.physbeh.2016.03.006] [Medline: 26969518]

5. Gillinov S, Etiwy M, Wang R, Blackburn G, Phelan D, Gillinov AM, et al. Variable accuracy of wearable heart rate monitors during aerobic exercise. Med Sci Sports Exerc 2017 Dec;49(8):1697-1703. [doi: 10.1249/MSS.0000000000001284] [Medline: 28709155]

6. Kroll RR, Boyd JG, Maslove DM. Accuracy of a wrist-worn wearable device for monitoring heart rates in hospital inpatients: a prospective observational study. J Med Internet Res 2016 Dec 20;18(9):e253 [FREE Full text] [doi: 10.2196/jmir.6025] [Medline: 27651304]

7. Shcherbina A, Mattsson CM, Waggott D, Salisbury H, Christle JW, Hastie T, et al. Accuracy in wrist-worn, sensor-based measurements of heart rate and energy expenditure in a diverse cohort. J Pers Med 2017 May 24;7(2) [FREE Full text] [doi: 10.3390/jpm7020003] [Medline: 28538708]

8. Wallen MP, Gomersall SR, Keating SE, Wisløff U, Coombes JS. Accuracy of heart rate watches: implications for weight management. PLoS One 2016;11(5):e0154420 [FREE Full text] [doi: 10.1371/journal.pone.0154420] [Medline: 27232714]

9. Wang R, Blackburn G, Desai M, Phelan D, Gillinov L, Houghtaling P, et al. Accuracy of wrist-worn heart rate monitors. JAMA Cardiol 2017 Jan 01;2(1):104-106. [doi: 10.1001/jamacardio.2016.3340] [Medline: 27732703]

10. Dooley EE, Golaszewski NM, Bartholomew JB. Estimating accuracy at exercise intensities: a comparative study of self-monitoring heart rate and physical activity wearable devices. JMIR Mhealth Uhealth 2017 Mar 16;5(3):e34 [FREE Full text] [doi: 10.2196/mhealth.7043] [Medline: 28302596]

11. Stahl SE, An HS, Dinkel DM, Noble JM, Lee JM. How accurate are the wrist-based heart rate monitors during walking and running activities? Are they accurate enough? BMJ Open Sport Exerc Med 2016;2(1):e000106 [FREE Full text] [doi: 10.1136/bmjsem-2015-000106] [Medline: 27900173]

12. El-Amrawy F, Nounou MI. Are currently available wearable devices for activity tracking and heart rate monitoring accurate, precise, and medically beneficial? Healthc Inform Res 2015 Oct;21(4):315-320 [FREE Full text] [doi: 10.4258/hir.2015.21.4.315] [Medline: 26618039]

13. Benedetto S, Caldato C, Bazzan E, Greenwood DC, Pensabene V, Actis P. Assessment of the Fitbit Charge 2 for monitoring heart rate. PLoS One 2018;13(2):e0192691 [FREE Full text] [doi: 10.1371/journal.pone.0192691] [Medline: 29489850]

14. Cadmus-Bertram L, Gangnon R, Wirkus EJ, Thraen-Borowski KM, Gorzelitz-Liebhauser J. Accuracy of heart rate monitoring by some wrist-worn activity trackers. Ann Intern Med 2017 Dec 17;167(8):607-608. [doi: 10.7326/L17-0380] [Medline: 29049770]

15. Jo E, Lewis K, Directo D, Kim MJ, Dolezal BA. Validation of biofeedback wearables for photoplethysmographic heart rate tracking. J Sports Sci Med 2016 Sep;15(3):540-547 [FREE Full text] [Medline: 27803634]

16. Spierer DK, Rosen Z, Litman LL, Fujii K. Validation of photoplethysmography as a method to detect heart rate during rest and exercise. J Med Eng Technol 2015;39(5):264-271. [doi: 10.3109/03091902.2015.1047536] [Medline: 26112379]

17. Bai Y, Hibbing P, Mantis C, Welk GJ. Comparative evaluation of heart rate-based monitors: Apple Watch vs Fitbit Charge HR. J Sports Sci 2018 Aug;36(15):1734-1741. [doi: 10.1080/02640414.2017.1412235] [Medline: 29210326] 
18. Parak J, Korhonen I. Evaluation of wearable consumer heart rate monitors based on photopletysmography. Conf Proc IEEE Eng Med Biol Soc 2014;2014:3670-3673. [doi: 10.1109/EMBC.2014.6944419] [Medline: 25570787]

19. Xie J, Wen D, Liang L, Jia Y, Gao L, Lei J. Evaluating the validity of current mainstream wearable devices in fitness tracking under various physical activities: comparative study. JMIR Mhealth Uhealth 2018 Apr 12;6(4):e94 [FREE Full text] [doi: 10.2196/mhealth.9754] [Medline: 29650506]

20. Sartor F, Papini G, Cox LG, Cleland J. Methodological shortcomings of wrist-worn heart rate monitors validations. J Med Internet Res 2018 Jul 02;20(7):e10108 [FREE Full text] [doi: 10.2196/10108] [Medline: 29967000]

21. Gorny AW, Liew SJ, Tan CS, Müller-Riemenschneider F. Fitbit Charge HR Wireless Heart Rate Monitor: validation study conducted under free-living conditions. JMIR Mhealth Uhealth 2017 Oct 20;5(10):e157 [FREE Full text] [doi: 10.2196/mhealth.8233] [Medline: 29055881]

22. Wilson K, Bell C, Wilson L, Witteman H. Agile research to complement agile development: a proposal for an mHealth research lifecycle. NPJ Digit Med 2018 Sep 13;1(1). [doi: 10.1038/s41746-018-0053-1]

23. Nelson BW. Open Science Framework.: Open Science Framework; 2018 Oct 12. Accuracy of the wrist-worn wearables during a 24-hour ecologically valid protocol URL: http://osf.io/6w2sh [accessed 2018-10-12] [WebCite Cache ID 737XKXN8R]

24. Fisher AJ, Medaglia JD, Jeronimus BF. Lack of group-to-individual generalizability is a threat to human subjects research. Proc Natl Acad Sci U S A 2018 Dec 03;115(27):E6106-E6115. [doi: 10.1073/pnas.1711978115] [Medline: 29915059]

25. Whitney RL, Ward DH, Marois MT, Schmid CH, Sim I, Kravitz RL. Patient perceptions of their own data in mHealth technology-enabled N-of-1 trials for chronic pain: qualitative study. JMIR Mhealth Uhealth 2018 Oct 11;6(10):e10291 [FREE Full text] [doi: 10.2196/10291] [Medline: 30309834]

26. Wichers M, Groot PC, Psychosystems, ESM Group, EWS Group. Critical slowing down as a personalized early warning signal for depression. Psychother Psychosom 2016;85(2):114-116 [FREE Full text] [doi: 10.1159/000441458] [Medline: 26821231]

27. Diaz KM, Thanataveerat A, Parsons FE, Yoon S, Cheung YK, Alcántara C, et al. The influence of daily stress on sedentary behavior: group and person ( $N$ of 1) level results of a 1-year observational study. Psychosom Med 2018 Sep;80(7):620-627. [doi: 10.1097/PSY.0000000000000610] [Medline: 29846309]

28. Sainsbury K, Vieira R, Walburn J, Sniehotta FF, Sarkany R, Weinman J, et al. Understanding and predicting a complex behavior using n-of-1 methods: photoprotection in xeroderma pigmentosum. Health Psychol 2018 Dec;37(12):1145-1158. [doi: 10.1037/hea0000673] [Medline: $\underline{\text { 30221970] }}$

29. Vahia IV, Sewell DD. Late-life depression: a role for accelerometer technology in diagnosis and management. Am J Psychiatry 2016 Aug 01;173(8):763-768. [doi: 10.1176/appi.ajp.2015.15081000] [Medline: 27477136]

30. Poldrack RA, Laumann TO, Koyejo O, Gregory B, Hover A, Chen MY, et al. Long-term neural and physiological phenotyping of a single human. Nat Commun 2015 Dec 09;6:8885 [FREE Full text] [doi: 10.1038/ncomms9885] [Medline: 26648521]

31. Chen R, Mias GI, Li-Pook-Than J, Jiang L, Lam HY, Chen R, et al. Personal omics profiling reveals dynamic molecular and medical phenotypes. Cell 2012 Mar 16;148(6):1293-1307 [FREE Full text] [doi: 10.1016/j.cell.2012.02.009] [Medline: 22424236]

32. de Geus EJ, Willemsen GH, Klaver CH, van Doornen LJ. Ambulatory measurement of respiratory sinus arrhythmia and respiration rate. Biol Psychol 1995 Nov 16;41(3):205-227. [Medline: 8608201]

33. Willemsen GH, De Geus EJ, Klaver CH, Van Doornen LJ, Carroll D. Ambulatory monitoring of the impedance cardiogram. Psychophysiology 1996 Mar;33(2):184-193. [Medline: $\underline{8851246]}$

34. Datta D. Github. 2018. AppleHealthAnalysis URL: https://github.com/deepankardatta/AppleHealthAnalysis [accessed 2018-10-12] [WebCite Cache ID 737XTtzDT]

35. Teramo N. Github. 2018. fitbitr URL: https://github.com/teramonagi/fitbitr [accessed 2018-10-12] [WebCite Cache ID 737Xb5IgE]

36. Nelson MB, Kaminsky LA, Dickin DC, Montoye AH. Validity of consumer-based physical activity monitors for specific activity types. Med Sci Sports Exerc 2016 Dec;48(8):1619-1628. [doi: 10.1249/MSS.0000000000000933] [Medline: 27015387]

37. Bunn JA, Navalta JW, Fountaine CJ, Reece JD. Current state of commercial wearable technology in physical activity monitoring 2015-2017. Int J Exerc Sci 2018;11(7):503-515 [FREE Full text] [Medline: 29541338]

38. Consumer Technology Association. 2018. Physical Activity Monitoring for Heart Rate ANSI/CTA-2065 URL: https:/ /standards.cta.tech/kwspub/published docs/CTA-2065-Preview.pdf [accessed 2018-12-02] [WebCite Cache ID 74MLno6Qd]

39. US Department of Health and Human Services, Food and Drug Administration, Center for Drug Evaluation and Research (CDER). Food and Drug Administration. 2006. Guidance for Industry, Investigating Out-of-Specification (OOS), Test Results for Pharmaceutical Production URL: https://www.fda.gov/downloads/drugs/guidances/ucm070287.pdf [accessed 2018-12-02] [WebCite Cache ID 74MMFRgva]

40. Rosenberger ME, Buman MP, Haskell WL, McConnell MV, Carstensen LL. Twenty-four hours of sleep, sedentary behavior, and physical activity with nine wearable devices. Med Sci Sports Exerc 2016 Mar;48(3):457-465 [FREE Full text] [doi: 10.1249/MSS.0000000000000778] [Medline: 26484953] 
41. Fokkema T, Kooiman TJ, Krijnen WP, VAN DER Schans CP, DE Groot M. Reliability and validity of ten consumer activity trackers depend on walking speed. Med Sci Sports Exerc 2017 Dec;49(4):793-800. [doi: 10.1249/MSS.0000000000001146] [Medline: 28319983]

42. Datta D. Github. 2018. blandr: a Bland-Altman Method Comparison package for R URL: https://github.com/deepankardatta/ blandr/ [accessed 2018-10-12] [WebCite Cache ID 737XhtKwL]

43. Lehnert B. The Comprehensive R Archive Network. Package 'BlandAltmanLeh' URL: https://cran.r-project.org/web/ packages/BlandAltmanLeh/BlandAltmanLeh.pdf [accessed 2018-10-12] [WebCite Cache ID 737XpMktd]

44. Zaki R, Bulgiba A, Ismail R, Ismail NA. Statistical methods used to test for agreement of medical instruments measuring continuous variables in method comparison studies: a systematic review. PLoS One 2012;7(5):e37908 [FREE Full text] [doi: 10.1371/journal.pone.0037908] [Medline: 22662248]

45. Bland JM, Altman DG. Statistical methods for assessing agreement between two methods of clinical measurement. Lancet 1986 Feb 08;1(8476):307-310. [Medline: 2868172]

46. Signorell A. The Comprehensive R Archive Network. 2018. DescTools: Tools for Descriptive Statistics URL: https://cran. r-project.org/web/packages/DescTools/index.html [accessed 2018-10-12] [WebCite Cache ID 737XwKTDx]

47. Terry N, Wiley LF. Liability for mobile health and wearable technologies. Ann Health Law 2016 Feb 02 [FREE Full text]

48. Vandewalle G, Middleton B, Rajaratnam SM, Stone BM, Thorleifsdottir B, Arendt J, et al. Robust circadian rhythm in heart rate and its variability: influence of exogenous melatonin and photoperiod. J Sleep Res 2007 Jun;16(2):148-155 [FREE Full text] [doi: 10.1111/j.1365-2869.2007.00581.x] [Medline: 17542944]

49. Gupta A, Shetty H. Circadian variation in stroke - a prospective hospital-based study. Int J Clin Pract 2005 Nov;59(11):1272-1275. [doi: 10.1111/j.1368-5031.2005.00678.x] [Medline: 16236079]
Abbreviations
ADL: activities of daily living
bpm: beats per minute
CCC: concordance class correlation
ECG: electrocardiogram
HR: heart rate
LoA: limit of agreement
MAE: mean absolute error
MAPE: mean absolute percent error
ME: mean error
OSF: Open Science Framework
PPG: photoplethysmography

Edited by G Eysenbach; submitted 19.04.18; peer-reviewed by A Shcherbina, J Quiroz, M Lang, P Innominato; comments to author
25.08.18; revised version received 12.10.18; accepted 10.11.18; published 11.03.19
Please cite as:
Nelson BW, Allen NB
Accuracy of Consumer Wearable Heart Rate Measurement During an Ecologically Valid 24-Hour Period: Intraindividual Validation
Study
JMIR Mhealth Uhealth 2019;7(3):e10828
URL: $\underline{\text { https://mhealth.jmir.org/2019/3/e10828/ }}$
doi: $\underline{10.2196 / 10828}$
PMID: $\underline{30855232}$

CBenjamin W Nelson, Nicholas B Allen. Originally published in JMIR Mhealth and Uhealth (http://mhealth.jmir.org), 11.03.2019. This is an open-access article distributed under the terms of the Creative Commons Attribution License (https://creativecommons.org/licenses/by/4.0/), which permits unrestricted use, distribution, and reproduction in any medium, provided the original work, first published in JMIR mhealth and uhealth, is properly cited. The complete bibliographic information, a link to the original publication on http://mhealth.jmir.org/, as well as this copyright and license information must be included. 